\title{
Large deviation functions in a system of diffusing particles with creation and annihilation
}

\author{
V. Popkov ${ }^{1,3}$ and G. M. Schütz $z^{2,3, *}$ \\ ${ }^{1}$ Dipartimento di Fisica “E.R. Caianiello” and Consorzio Nazionale Interuniversitario per le Scienze Fisiche della Materia (CNISM), \\ Università di Salerno, 84084 Fisciano, Italy \\ ${ }^{2}$ Theoretical Soft Matter and Biophysics, Institute of Complex Systems, Forschungszentrum Jülich, D-52428 Jülich, Germany \\ ${ }^{3}$ Interdisziplinäres Zentrum für Komplexe Systeme, University of Bonn, 53115 Bonn, Germany
}

(Received 1 June 2011; published 19 August 2011)

\begin{abstract}
Large deviation functions for an exactly solvable lattice gas model of diffusing particles on a ring, subject to pair annihilation and creation, are obtained analytically using exact free-fermion techniques. Our findings for the large deviation function for the current are compared to recent results of Appert-Rolland et al. [Phys. Rev. E 78,021122 (2008)] for diffusive systems with conserved particle number. Unlike conservative dynamics, our nonconservative model has no universal finite-size corrections for the cumulants. However, the leading Gaussian part has the same variance as in the conservative case. We also elucidate some properties of the large deviation functions associated with particle creation and annihilation.
\end{abstract}

DOI: 10.1103/PhysRevE.84.021131

PACS number(s): 05.40.-a, 02.50.Ga, 64.60.De, 05.70.Ln

\section{INTRODUCTION}

The time-integrated particle current $J_{t}$ in a stochastic interacting particle system is a fluctuating quantity whose distribution has been studied intensely in the last decade and has turned to fall into distinct universality classes and also to exhibit interesting mathematical behavior which has been elucidated in some detail particularly for driven systems (see, e.g., [1-6]). Recently Appert-Rolland et al. [7] have studied finite-size effects of the cumulants for the symmetric simple exclusion process (SSEP) on a ring of $L$ sites. Here the net current is zero on average, but using Bethe ansatz they found for the asymptotic cumulant function (i) a leading Gaussian behavior with a variance $\kappa L$ proportional to system size and coefficient given by the compressibility $\kappa$ of the stationary particle system, and (ii) a universal finite-size correction of the form $L^{-2} D \mathcal{F}\left[\kappa \kappa^{\prime \prime} L^{2} s^{2} /\left(4 D^{2}\right)\right]$ where $D$ is the collective diffusion coefficient and $s$ is the intensive variable conjugate to the current. They also derived the same result using the macroscopic fluctuation theory by Bertini et al. [8] which suggests that this behavior is universal for all diffusive particle systems on a ring with one conserved type of particles. As pointed out in [7] the universal scaling form arises from the discreteness of the wave vectors of the fluctuating modes on the ring.

The existence of a single conservation law for the particles is indeed crucial for the theory. Even though the macroscopic fluctuation theory has recently been extended to reactiondiffusion systems where the particle number is not conserved [9], nothing is known about the cumulant function in finite particle systems. Here we argue that in the absence of a conservation law the Gaussian part in the cumulant function survives (with some a priori unknown coefficient) and that the universal finite-size corrections disappear since the nonconservative dynamics destroy the fluctuation modes exponentially fast in time. By considering an exactly solvable reaction-diffusion process, the SSEP with annihilation and creation of pairs, we confirm in this work this picture in a mathematically rigorous

\footnotetext{
*g.schuetz@fz-juelich.de
}

fashion. We also compute the coefficient of the Gaussian part which turns out to be again the compressibility $\kappa$. Besides this abstract interest our study is also motivated by the fact that nonconservative dynamics arise in several applications of stochastic interacting particle systems to soft matter systems and biological processes, in particular for modeling molecular motors in the case of driven systems [10,11] and relaxation of entangled polymers in the case of the SSEP [12]. Going beyond the current distribution we consider also various other large deviation functions associated with the stochastic dynamics of the process.

The plan of the paper is the following. In Sec. II we define our model and review some properties that we need in Sec. III for the computation of the cumulant function of the current. In Sec. IV we study other large deviation functions related to the reactivity of the process and in Sec. V we draw some conclusions and propose open questions.

\section{SSEP WITH PAIR CREATION AND ANNIHILATION}

\section{A. Definition and spectral properties}

The symmetric simple exclusion process $[13,14]$ is a Markov process where particles jump randomly on a lattice $\Lambda$ subject to the exclusion rule that each lattice site is occupied by at most one particle. Therefore the instantaneous state $\eta_{t}$ of the system at time $t$ can be represented by occupation numbers $\eta_{t}(k)$ for lattice sites $k \in \Lambda$. We shall consider the one-dimensional period lattice of $L$ sites where $L$ is even. Particle jumps from a site $k$ occur independently after an exponentially distributed random time with mean $\tau=1 /(2 D)$ with equal probability to one of the nearest neighbor sites $k \pm 1$. In addition, we allow for random creation and annihilation of neighboring particle pairs also total rate $2 D$ such that an existing particle pair is annihilated with rate $\lambda$ while a particle pair may be created on neighboring empty sites with rate $\mu=2 D-\lambda$. Stationary and dynamical properties of this process have been studied in detail some time ago (see, e.g., [14-16] for a review and mappings to other processes). Here we go beyond this work by also studying the integrated current $J(t)$ and reactivities $R^{ \pm}(t)$ of the process. 
The integrated current is the total net number of particle jumps in the system up to time $t$ while $R^{+}(t)\left[R^{-}(t)\right]$ is the total number of creation (annihilation) events up to time $t$.

The particle dynamics are governed by the Master equation which in quantum Hamiltonian form [14] can be written

$$
\frac{\partial|P(t)\rangle}{\partial t}=-H|P(t)\rangle \text {. }
$$

Here the probability distribution $P(\eta, t)$ is represented by the probability vector

$$
|P(t)\rangle \sum_{\eta} P(\boldsymbol{\eta}, t)|\boldsymbol{\eta}\rangle\langle\boldsymbol{\eta}|,
$$

where $|\boldsymbol{\eta}\rangle=|\eta(1)\rangle \otimes \cdots \otimes|\eta(L)\rangle$ and the dual vectors $\langle\boldsymbol{\eta}|$ form a tensor basis with scalar product $\left\langle\boldsymbol{\eta}^{\prime} \mid \boldsymbol{\eta}\right\rangle=\delta_{\boldsymbol{\eta}^{\prime}, \boldsymbol{\eta}}$. In our choice of basis

$$
|0\rangle=\left(\begin{array}{l}
1 \\
0
\end{array}\right), \quad|1\rangle=\left(\begin{array}{l}
0 \\
1
\end{array}\right)
$$

represents a vacant site or a occupied site, respectively. The generator

$$
\begin{aligned}
H= & -\sum_{k=1}^{L}\left[D\left(\sigma_{k}^{+} \sigma_{k+1}^{-}-n_{k} v_{k+1}\right)+D\left(\sigma_{k}^{-} \sigma_{k+1}^{+}-v_{k} n_{k+1}\right)\right. \\
& \left.+\lambda\left(\sigma_{k}^{+} \sigma_{k+1}^{+}-n_{k} n_{k+1}\right)+\mu\left(\sigma_{k}^{-} \sigma_{k+1}^{-}-v_{k} v_{k+1}\right)\right]
\end{aligned}
$$

encodes the stochastic dynamics with transition rates between configurations as off-diagonal matrix elements and the inverse lifetimes (exit rates) of the configurations on the diagonal. Here we use the Pauli matrices $\sigma_{k}^{x, y, z}$ acting on site $k$ to construct the spin-1/2 ladder operators

$$
\begin{gathered}
\sigma_{k}^{+}=\frac{1}{2}\left(\sigma_{k}^{x}+\sigma_{k}^{y}\right)=\left(\begin{array}{ll}
0 & 1 \\
0 & 0
\end{array}\right)_{k}, \\
\sigma_{k}^{-}=\frac{1}{2}\left(\sigma_{k}^{x}-\sigma_{k}^{y}\right)=\left(\begin{array}{ll}
0 & 0 \\
1 & 0
\end{array}\right)_{k}
\end{gathered}
$$

which annihilate $\left(\sigma_{k}^{+}\right)$or create $\left(\sigma_{k}^{-}\right)$particles. This somewhat counterintuitive notation goes back to the standard choice of basis in the condensed matter literature on quantum spin chains. Furthermore,

$$
n_{k}=\frac{1}{2}\left(1-\sigma_{k}^{z}\right)=\left(\begin{array}{ll}
0 & 0 \\
0 & 1
\end{array}\right)_{k}
$$

is the particle number operator while $v_{k}=1-n_{k}$ projects on vacant sites.

The generator $H$ describes diffusive hopping with rate $D$, and annihilation and creation of pairs with rates $\lambda$ and $\mu$, respectively. For the choice

$$
\lambda+\mu=2 D
$$

the terms bilinear in the $n_{k}$ vanish in the stochastic Hamiltonian (4) and it becomes a free fermion one [14,15],

$$
\begin{aligned}
H= & -\sum_{k=1}^{L}\left[D\left(\sigma_{k}^{+} \sigma_{k+1}^{-}+\sigma_{k}^{-} \sigma_{k+1}^{+}\right)+\lambda \sigma_{k}^{+} \sigma_{k+1}^{+}+\mu \sigma_{k}^{-} \sigma_{k+1}^{-}\right] \\
& +(2 D-2 \mu) \sum_{k=1}^{L} n_{k}+\mu L .
\end{aligned}
$$

To diagonalize $H$ we follow the approach of [17]. By consecutive application of the Jordan-Wigner transformation and Fourier transform, and introducing bilinear operators $B_{p}^{ \pm}, N_{p}$ which satisfy the same algebra as the spin-1/2 ladder operators $\sigma_{p}^{ \pm}, N_{p}$ (see [14], Chap. 9 for details) we obtain

$$
\begin{aligned}
H= & -\sum_{p>0}\left[\left(4 D \cos q_{p}\right) N_{p}+2 \sin q_{p}\left(\lambda B_{p}+\mu B_{p}^{+}\right)\right] \\
& +2(\lambda-\mu) \sum_{p>0} N_{p}+\mu L .
\end{aligned}
$$

Here $q_{p}=2 \pi p / L$, and $p$ takes values $p=\frac{1}{2}, \frac{3}{2}, \ldots, \frac{L-1}{2}$. This choice of $p$ corresponds to the sector with an even number of particles. Using the representation of

$$
B_{p}=\left(\begin{array}{ll}
0 & 1 \\
0 & 0
\end{array}\right)_{p}, \quad N_{p}=\left(\begin{array}{ll}
0 & 0 \\
0 & 1
\end{array}\right)_{p}
$$

we finally obtain $H=\sum_{p>0} H_{p}$ with

$$
H_{p}=\left(\begin{array}{cc}
2 \mu & -2 \lambda \sin q_{p} \\
-2 \mu \sin q_{p} & 2 \lambda-4 D \cos q_{p}
\end{array}\right)_{p} .
$$

It is straightforward to find that the minimal eigenvalue $\varepsilon_{p}$ of $H_{p}$ is $\min \varepsilon_{p}=-2 \mu \cos q_{p}$. The minimal eigenvalue $\varepsilon$ of the whole Hamiltonian is then given by

$$
\varepsilon=\sum_{p>0} \varepsilon_{p}=0,
$$

in accordance with the stochasticity of $H$.

\section{B. Cumulant functions}

So far we have considered only the dynamics of the particles in terms of the configurations $\boldsymbol{\eta}_{t}$. In order to keep track of the current we need to introduce another stochastic variable $J(t)$ which counts the total number of particle jumps to the right (clockwise on the ring) minus the total number of jumps to the left (counterclockwise) during the evolution of the process until time $t$. The cumulant function of the current $J(t)$ is then defined by

$$
\begin{aligned}
\Psi(s, t) & =\ln \left\langle e^{s J(t)}\right\rangle=\ln \sum_{J} P[J(t)] e^{s J(t)} \\
& =\ln \sum_{J} e^{t f(J / t=j)} e^{s J(t)}
\end{aligned}
$$

where $P[J(t)]$ is the probability distribution of the current which takes for large $t$ the large deviation form $P[J(t)] \sim$ $\exp t f(j)$ with the large deviation function $f(j)$ for the mean current $j=J(t) / t$. As $t \rightarrow \infty$ one obtains [18,19]

$$
\mu(s) \equiv \lim _{t \rightarrow \infty} \Psi(s, t) / t=-\min _{i} \Lambda_{i}(s),
$$

where $\min _{i} \Lambda_{i}(s)$ is the lowest eigenvalue of the modified Hamiltonian

$$
H(s)=e^{s} H_{+}+e^{-s} H_{-}+H_{0}
$$

in which $H_{+}$is the part of the original Hamiltonian that corresponds to the moves to the right ( $\sigma_{k}^{+} \sigma_{k+1}^{-}$terms), $H_{-}$ is the part of the original Hamiltonian that corresponds to 
moves to the left $\left(\sigma_{k}^{-} \sigma_{k+1}^{+}\right.$terms), and $H_{0}$ is the remaining part in $H=H_{+}+H_{-}+H_{0}$. With the approach of [17] it is straightforward to verify that $H(s)=\sum_{p>0} H_{p}(s)$, where

$$
H_{p}(s)=\left(\begin{array}{cc}
2 \mu & -2 \lambda \sin q_{p} \\
-2 \mu \sin q_{p} & 2 \lambda-4 D \cosh s \cos q_{p}
\end{array}\right)_{p} .
$$

We remark that since we are considering a particle system with finite state space, the asymptotic large deviation function does not depend on the initial configuration of the particle system. Hence discontinuities such as those reported for the zero-range process $[20,21]$ cannot occur.

In a similar fashion one can analyze other dynamical properties associated with the stochastic reaction-diffusion dynamics. The total jump activity $K(t)$ counts the total number of jumps during the evolution up to time $t$, irrespective of jump direction. Introducing in analogy to (13) the cumulant function with conjugate variable $x$ one obtains its asymptotic form for large times

$$
\mu^{K}(x)=-\min _{i} \Lambda_{i}^{K}(x),
$$

where $\min _{i} \Lambda_{i}^{K}(x)$ is the lowest eigenvalue of the modified Hamiltonian

$$
H^{K}(x)=e^{x}\left(H_{+}+H_{-}\right)+H_{0}=\sum_{p} H_{p}^{K}(x) .
$$

Here

$$
H_{p}^{K}(x)=\left(\begin{array}{cc}
2 \mu & -2 \lambda \sin q_{p} \\
-2 \mu \sin q_{p} & 2 \lambda-4 D \mathrm{e}^{x} \cos q_{p}
\end{array}\right)_{p}
$$

Generally we can count right and left jumps separately to obtain the associated individual integrated currents $J^{ \pm}(t)$ and keep track also the number $R^{ \pm}(t)$ of creation and annihilation events. This gives rise to joint cumulant functions $\Psi\left(s_{+}, s_{-}, r_{+}, r_{-}, t\right)$ whose asymptotic form $\mu\left(s_{+}, s_{-}, r_{+}, r_{-}\right)$is given by the lowest eigenvalue of the matrix $H\left(s_{+}, s_{-}, r_{+}, r_{-}\right)$ which is a sum of the matrices

$$
\begin{aligned}
& H_{p}\left(s_{+}, s_{-}, r_{+}, r_{-}\right) \\
& \quad=\left(\begin{array}{cc}
2 \mu & -2 \lambda \mathbf{e}^{r_{-}} \sin q_{p} \\
-2 \mu \mathbf{e}^{r_{+}} \sin q_{p} & 2 \lambda-2 D\left(\mathbf{e}^{s_{-}}+\mathbf{e}^{s_{+}}\right) \cos q_{p}
\end{array}\right)_{p} .
\end{aligned}
$$

For the large deviation function already defined one has $\mu(s)=$ $\mu(s,-s, 0,0)$ and $\mu^{K}(x)=\mu(x, x, 0,0)$.

\section{LARGE DEVIATION FUNCTION FOR THE CURRENT}

The $n$-order cumulants $C_{n}$ of the current are the coefficients of Taylor series

$$
\mu(s)=\sum_{n=0}^{\infty} C_{n} s^{n}
$$

Since the jumps are symmetric we trivially have $\mu(s)=\mu(-s)$ and therefore all odd cumulants vanish. Also trivially $C_{0}=$ 1. Furthermore, if either $\lambda=0$ or $\mu=0$ then the system approaches an absorbing state (completely full or completely empty lattice, respectively) after a finite time of order $L^{2}$. Therefore the integrated current reaches a finite value and the asymptotic cumulant function $\mu(s)$ is identically zero.

In order to compute the nontrivial cumulants $C_{2 n}$ with $n \geqslant 1$ we restrict ourselves to the generic case $\lambda, \mu \neq 0$. Diagonalizing (15) using the representation (16) we find

$$
\varepsilon_{p}=2 D\left(1-\cosh s \cos q_{p}\right)-\sqrt{\left[2 D-(\lambda-\mu) \cosh s \cos q_{p}\right]^{2}+4 \lambda \mu \sinh ^{2} s \cos ^{2} q_{p}} .
$$

Hence, in order to compute the cumulants we have to make a Taylor expansion in $s$ of the series

$$
\begin{gathered}
\mu(s)=\sum_{p>0} 2 D\left(\cosh s \cos q_{p}-1\right)+\sqrt{\left[2 D-(\lambda-\mu) \cosh s \cos q_{p}\right]^{2}+4 \lambda \mu \sinh ^{2} s \cos ^{2} q_{p},} \\
\mu(s)=\sum_{p>0}\left\{-2 D+\sqrt{\left.\left[2 D-(\lambda-\mu) \cosh s \cos q_{p}\right]^{2}+4 \lambda \mu \sinh ^{2} s \cos ^{2} q_{p}\right\}}\right.
\end{gathered}
$$

since $\sum_{p>0} \cos q_{p}=0$. From this sum we obtain the cumulants through the derivatives

$$
C_{2 n}=(2 n) !{\frac{\partial^{2 n} \mu(s)}{\partial s^{2 n}}}_{s=0}
$$

We study first the variance $C_{2}$. For large system size we define $c_{2}=\lim _{L \rightarrow \infty} C_{2} / L$ and expand

$$
\tilde{\mu}(s)=\lim _{L \rightarrow \infty} \mu(s) / L=-\frac{1}{2 \pi} \int_{0}^{\pi} d q\left\{2 D-\sqrt{[2 D-(\lambda-\mu) \cosh s \cos q]^{2}+4 \lambda \mu \sinh ^{2} s \cos ^{2} q}\right\}
$$

in $s$. With

$$
f(s, q)=\left\{2 D-\sqrt{[2 D-(\lambda-\mu) \cosh s \cos q]^{2}+4 \lambda \mu \sinh ^{2} s \cos ^{2} q}\right\}
$$


one has

$$
f(s, q)=s^{2} \frac{2 \lambda \mu \cos ^{2} q}{2 D-(\lambda-\mu) \cos q}+O\left(s^{4}\right) .
$$

Using the identity

$$
I(A)=\int_{0}^{\pi} \frac{\cos ^{2} q}{1-A \cos q} d q=\frac{\pi}{A^{2}}\left(-1+\frac{1}{\sqrt{1-A^{2}}}\right),
$$

which is valid for $|A|<1$, and taking into account $2 D=$ $\lambda+\mu$, we obtain after some algebra

$$
I\left(\frac{\lambda-\mu}{\lambda+\mu}\right)=\pi \frac{(\lambda+\mu)^{2}}{2 \sqrt{\lambda \mu}(\sqrt{\lambda}+\sqrt{\mu})^{2}} .
$$

Hence

$$
c_{2}=\frac{1}{2} \frac{\sqrt{\lambda \mu}(\lambda+\mu)}{(\sqrt{\lambda}+\sqrt{\mu})^{2}} .
$$

On the other hand, the stationary density of the annihilationcreation process is given by [15]

$$
\rho_{\text {stat }}=\frac{\sqrt{\mu}}{\sqrt{\lambda}+\sqrt{\mu}} .
$$

Using this we find for large $L$,

$$
\begin{aligned}
C_{2} & =\frac{\left\langle J^{2}\right\rangle-\langle J\rangle^{2}}{t}=\left[\frac{\partial^{2} \mu(s)}{\partial s^{2}}\right]_{s=0} \\
& =\frac{\sqrt{\lambda \mu}(\lambda+\mu)}{(\sqrt{\lambda}+\sqrt{\mu})^{2}} L=2 D \kappa(\rho) L,
\end{aligned}
$$

where

$$
\kappa(\rho):=\frac{\left\langle N^{2}\right\rangle-\langle N\rangle^{2}}{L}=\rho(1-\rho)
$$

is the stationary compressibility of the system, measuring the fluctuation of the particle number per unit volume. Formula (33) is in agreement with the theory developed in [7] for diffusive systems with a conserved number of particles.

Next we consider finite-size corrections and compare our results with the scaling prediction of Ref. [7]

$$
\frac{\left\langle Q^{2 n}\right\rangle_{c}}{t}=\left.\frac{\partial^{2 n} \mu(s)}{\partial s^{2 n}}\right|_{s=0}=\frac{(2 n) ! B_{2 n-2}}{2^{n}(n-1) ! n !} \sigma^{n} L^{2 n-2}
$$

for higher order cumulants $n \geqslant 2$ which is equivalent to

$$
\begin{aligned}
\lim _{L \rightarrow \infty} L^{2}\left[\mu(s)-\frac{s^{2}}{2} \mu^{\prime \prime}(0)\right] & =\lim _{L \rightarrow \infty} L^{2}\left\{\sum_{n=2}^{\infty} \frac{\left[\frac{\partial^{2 n} \mu(s)}{\partial s^{2 n}}\right]_{s=0}}{(2 n) !} s^{2 n}\right\} \\
& =\Phi\left[-\frac{\sigma(\rho)}{4} L^{2} s^{2}\right]
\end{aligned}
$$

where $\sigma(\rho)=2 \kappa(\rho)$ and

$$
\begin{aligned}
\Phi(u) & =-4 \sum_{n \geqslant 1}\left(n \pi \sqrt{n^{2} \pi^{2}-2 u}-n^{2} \pi^{2}+u\right) \\
& =\frac{1}{3} u^{2}+\frac{1}{45} u^{3}+\frac{1}{378} u^{4}+O\left(u^{5}\right)
\end{aligned}
$$

is a universal scaling function.

In order to find the fourth and higher order current cumulants, we need higher order terms in the expansion of $\mu(s)$. Using the Euler-Maclaurin summation formula the sum
(24) can be approximated by to leading order by the integral $\frac{L}{2 \pi} \int_{0}^{\pi} \cdots$. The subleading Euler-Maclaurin terms give a power series in $L^{-k}$. The expression inside the square root in the integral can be expanded in $s$ as follows:

$$
\begin{aligned}
\mathbf{D} & =[2 D-(\lambda-\mu) \cos q \cosh s]^{2}+4 \lambda \mu \cos ^{2} q \sinh ^{2} s \\
& =\sum_{k=0}^{\infty} A_{2 k} \frac{s^{2 k}}{(2 k) !},
\end{aligned}
$$

where

$$
\begin{array}{r}
A_{0}=[2 D+(\mu-\lambda) \cos q]^{2}, \\
A_{2 k}=4 D \cos q\left[2^{2 k-2}(2 D \cos q)+\mu-\lambda\right] \text { for } \quad k>0 .
\end{array}
$$

This yields

$$
\left.\frac{\partial^{4} \sqrt{\mathbf{D}}}{\partial s^{4}}\right|_{s=0}=\frac{A_{4}}{2 A_{0}^{1 / 2}}-\frac{3\left(A_{2}\right)^{2}}{4 A_{0}^{3 / 2}}
$$

and

$$
\left.\frac{\partial^{4} \mu(s)}{\partial s^{4}}\right|_{s=0}=\sum_{p>0}\left[\frac{A_{4}}{2 A_{0}^{1 / 2}}-\frac{3\left(A_{2}\right)^{2}}{4 A_{0}^{3 / 2}}\right] .
$$

It is easy to compute this expression for $\lambda=\mu=D$, in which case we have

$$
\begin{aligned}
\left.\frac{\partial^{4} \mu(s)}{\partial s^{4}}\right|_{s=0, \lambda=\mu} & =\sum_{p>0} 8 D \cos ^{2} q-6 D \cos ^{4} q \\
& \approx \frac{7}{8} L D+O(1),
\end{aligned}
$$

where $O(1)$ comes from the Euler-Maclaurin expansion. We see that the fourth cumulant is proportional to $L$, rather than $L^{2}$ as predicted by (35). Computing higher order cumulants for the case $\lambda=\mu=D$ is straightforward, since only integrals $\int_{0}^{\pi} \cos ^{2 n} x d x=\sqrt{\pi} \Gamma(n+1 / 2) / n$ ! are involved. In particular, we obtain for $n=3,4,5$

$$
\begin{aligned}
\left.\frac{\partial^{6} \mu(s)}{\partial s^{6}}\right|_{s=0, \lambda=\mu} & =-\frac{7}{16} L D+O(1), \\
\left.\frac{\partial^{8} \mu(s)}{\partial s^{8}}\right|_{s=0, \lambda=\mu} & =\frac{1387}{128} L D+O(1), \\
\left.\frac{\partial^{10} \mu(s)}{\partial s^{10}}\right|_{s=0, \lambda=\mu} & =-\frac{45577}{256} L D+O(1) .
\end{aligned}
$$

We see that $\left.\frac{\partial^{2 n} \mu(s)}{\partial s^{2 n}}\right|_{s=0} \sim L$, instead of being proportional to $L^{2 n-2}$, predicted in [7]. From the structure of (24) it is clear that this fact generalizes to the case $\lambda \neq \mu$. In other words, the scaling function $\Phi$, defined by (36) vanishes identically: $\lim _{L \rightarrow \infty} \Phi\left(L^{2} s^{2}\right)=0$. 


\section{LARGE DEVIATION FUNCTION OF THE REACTION PROCESSES}

Diagonalizing $H\left(s_{+}, s_{-}, r_{+}, r_{-}\right)$using the representation (20) we find

$$
\varepsilon_{p}=D\left(2-\left(\mathrm{e}^{s_{+}}+\mathrm{e}^{s_{-}}\right) \cos q_{p}\right)-\sqrt{\left[\mu-\lambda+D\left(\mathrm{e}^{s_{+}}+\mathrm{e}^{s_{-}}\right) \cos q_{p}\right]^{2}+4 \lambda \mu \mathrm{e}^{r_{+}+r_{-}} \sin ^{2} q_{p}}
$$

This leads to the general large deviation function

$$
\mu\left(s_{+}, s_{-}, r_{+}, r_{-}\right)=\sum_{p>0}\left(-2 D+\sqrt{\left[\mu-\lambda+D\left(\mathrm{e}^{s_{+}}+\mathrm{e}^{s_{-}}\right) \cos q_{p}\right]^{2}+4 \lambda \mu \mathrm{e}^{r_{+}+r_{-}} \sin ^{2} q_{p}}\right) .
$$

Below we consider some special cases.

\section{A. Pair annihilation}

First we focus on the large deviation function for annihilation events up to time $t$, that is, we consider $s_{+}=$ $s_{-}+r_{-}=0$. For notational simplicity we set $r_{+}=\alpha$ and $\nu(\alpha)=\mu(0,0,0, \alpha)$. After a little algebra we obtain from (48)

$$
v(\alpha)=\sum_{p>0}[-2 D+\sqrt{\mathbf{D}(\alpha)}]
$$

where $\mathbf{D}(\alpha)=[2 D+(\mu-\lambda) \cos q]^{2}+4 \lambda \mu\left(e^{\alpha}-1\right) \sin ^{2} q$.

The structure of this expression shows that all cumulants are proportional to system size. We compute explicitly the first and second cumulant, that is, mean and variance of the distribution. To this end we expand $\mathbf{D}(\alpha)=\sum_{k=0}^{\infty} A_{k} \frac{\alpha^{k}}{(k) !}$ where

$$
\begin{aligned}
& A_{0}=[2 D+(\mu-\lambda) \cos q]^{2}, \\
& A_{k}=4 \lambda \mu \sin ^{2} q \text { for } k>0 .
\end{aligned}
$$

Note that all $A_{k}$ for $k>0$ are equal. Then we get

$$
\begin{aligned}
\left.v^{\prime}(\alpha)\right|_{\alpha=0} & =\sum_{p} \frac{A_{1}}{2 A_{0}^{1 / 2}}=\sum_{p} \frac{\lambda \mu}{D} \frac{\sin ^{2} q}{1+K \cos q}, \\
\left.v^{\prime \prime}(\alpha)\right|_{\alpha=0} & =\sum_{p} \frac{A_{2}}{2 A_{0}^{1 / 2}}-\frac{1}{4} \frac{\left(A_{1}\right)^{2}}{A_{0}^{3 / 2}},
\end{aligned}
$$

where $K=(\mu-\lambda) /(\mu+\lambda)$. Using the identity

$$
\int_{0}^{\pi} \frac{\sin ^{2} q}{1+K \cos q} d q=\frac{\pi}{A^{2}}\left(1-\sqrt{1-A^{2}}\right),
$$

we obtain

$$
\left.v^{\prime}(\alpha)\right|_{\alpha=0}=\sqrt{\lambda \mu} \kappa(\rho) L=2 D \frac{\rho^{2}(1-\rho)^{2}}{\rho^{2}+(1-\rho)^{2}} L .
$$

Here we have used $\sqrt{\lambda \mu}=2 D \rho(1-\rho) /\left[\rho^{2}+(1-\rho)^{2}\right]$. In particular, for $\lambda=\mu=D$ we have $\left.\nu^{\prime}(\alpha)\right|_{\alpha=0}=D L / 4$.

In order to compute the second cumulant we use the fact that all $A_{k}$ are equal. Therefore we can write

$$
\begin{aligned}
v^{\prime \prime}(0) & =v^{\prime}(0)-\sum_{p} \frac{1}{4} \frac{\left(A_{1}\right)^{2}}{A_{0}^{3 / 2}} \\
& \approx \mu^{\prime}(0)-\frac{\lambda^{2} \mu^{2}}{2 D^{3}} \frac{L}{2 \pi} \int_{0}^{\pi} \frac{\sin ^{4} q}{(1+K \cos q)^{3}} .
\end{aligned}
$$

Using the identity

$$
\int_{0}^{\pi} \frac{\sin ^{4} q}{(1+A \cos q)^{3}}=I(A)=\frac{3 \pi}{A^{4}} \frac{\left(1-\sqrt{1-A^{2}}\right)^{2}}{\sqrt{1-A^{2}}}
$$

and then $\left(1-\sqrt{1-K^{2}}\right) / K^{2}=2 w /(\sqrt{\mu}+\sqrt{\lambda})^{2}$, $\sqrt{1-A^{2}}=\sqrt{\lambda \mu} / w$, we obtain

$$
-\frac{\lambda^{2} \mu^{2}}{2 w^{3}} \frac{L}{2 \pi} \int_{0}^{\pi} \frac{\sin ^{4} q}{(1+K \cos q)^{3}}=-\frac{3}{2} \sqrt{\lambda \mu} \kappa^{2}(\rho) L
$$

where $\kappa(\rho)=\rho(1-\rho)=\sqrt{\lambda \mu} /(\sqrt{\mu}+\sqrt{\lambda})^{2}$. Using $\mu^{\prime}(0)=$ $\sqrt{\lambda \mu} \kappa(\rho) L$ we finally have

$$
\begin{aligned}
v^{\prime \prime}(0) & =\sqrt{\lambda \mu}\left[\kappa(\rho)-\frac{3}{2} \kappa^{2}(\rho)\right] L \\
& =2 D \frac{\kappa(\rho)^{2}}{\rho^{2}+(1-\rho)^{2}}\left[1-\frac{3}{2} \kappa(\rho)\right] L .
\end{aligned}
$$

\section{B. Pair creation and reaction balance}

We note that the cumulant function $\mu(0,0, \beta, 0)$ for the number of creation events is given by the same expression as for annihilation events. Indeed, for creation events we have to diagonalize

$$
\left(\begin{array}{cc}
2 \mu & -2 \lambda \sin q \\
-2 \mu e^{\beta} \sin q & 2 \lambda-4 D \cos q
\end{array}\right)
$$

which has the same spectrum as $H_{p}$ for annihilation events.

This property can be further elucidated by considering the cumulant function for the reaction balance, that is, the cumulant function $\mu(0,0,-\alpha, \alpha)$ for the difference between creation and annihilation events. One has to diagonalize

$$
\begin{aligned}
H_{p}(\alpha,-\alpha) & =\left(\begin{array}{cc}
2 \mu & -2 \lambda e^{\alpha} \sin q \\
-2 \mu e^{-\alpha} \sin q & 2 \lambda-4 D \cos q
\end{array}\right) \\
& =Q_{p}(\alpha) H_{p}(0,0) Q_{p}^{-1}(\alpha),
\end{aligned}
$$

where $Q_{p}=\exp \alpha n_{p}$. It follows immediately that the eigenvalues do not depend on $\alpha$. Hence, by the stochasticity property (12) one has $\mu(0,0,-\alpha, \alpha)=\mu(0,0,0,0)=0$.

At first sight it seems surprising that all cumulants of the reaction balance vanish. The origin of this property lies in the fact that we are considering a finite system with a bounded number of particles. For this reason the number of annihilations can deviate from the number of creations only by a finite amount. In 
the long time limit it follows from the similarity transformation in (57) that $\lim _{t \rightarrow \infty} \ln \left\langle\exp \left\{\alpha\left[R_{+}(t)-R_{-}(t)\right]\right\}\right\rangle=L \ln [(1-$ $\left.\left.\rho^{2}\right)+\rho^{2}+2 \rho(1-\rho) \cosh (\alpha / 2)\right]$.

\section{SOME CONCLUDING REMARKS}

We have argued that in a nonconservative reaction-diffusion system on a ring the universal finite-size corrections to the current cumulants which appear in conservative diffusive particle systems [7] should vanish since the uncorrelated reaction processes cause the diffusive relaxation modes to decay exponentially in time. This picture is well born out by the exactly solvable model of symmetric diffusion with pair annihilation and pair creation that we have analyzed in detail using exact free-fermion techniques. Hence there is an essential difference between current fluctuations in conservative and nonconservative single species systems. However, we also found that the fluctuations are Gaussian with a variance that has the same dependence on the static compressibility as derived for conservative diffusive systems. With regard to the fluctuation theory of [9] we remark that in that work the hopping is speeded up by a factor $L^{2}$ to obtain nontrivial hydrodynamics. The computation of finite-size corrections to the current cumulants for this case is an open problem.

We have also computed cumulants for the number of annihilation events which are all asymptotically proportional to system size. The long-time cumulants for the reaction balance, that is, of the distribution of the difference in the number of annihilation and creation events up to time $t \rightarrow \infty$ vanish. This has its simple explanation in the fact that we consider a finite system with finite local state space where the difference between the number of creation events and the number of annihilation events is strictly bounded. However, it is a simple exercise to show that the same is true for the zerorange process [22] if in addition to particle jumps one admits nonconservative dynamics with annihilation of single particle with a rate proportional to the particle jump rate and creation of single particle with an arbitrary rate. In this case the difference between annihilation and creation events is not bounded even on a finite lattice and the observation of vanishing cumulants becomes nontrivial. It would be interesting to understand whether these three features of our special model-Gaussian fluctuation of the integrated current with a variance given by the static compressibility, proportionality of all cumulants for the annilation process with system size, and vanishing cumulants for the reaction balance-are generic features of single-species reaction-diffusion systems on a ring.

\section{ACKNOWLEDGMENT}

This project was supported by Deutsche Forschungsgemeinschaft.
[1] K. Johansson, Commun. Math. Phys. 209, 437 (2000).

[2] M. Prähofer and H. Spohn, in In and Out of Equilibrium, Progress in Probability, Vol. 51, edited by V. Sidoravicius (Birkhäuser, Boston, MA, 2002), pp 185-204.

[3] A. Rákos and G. M. Schütz, J. Stat. Phys. 118, 511 (2005).

[4] A. Borodin. P. L. Ferrari, M. Prähofer, and T. Sasamoto, J. Stat. Phys. 129, 1055 (2007).

[5] C. A. Tracy and H. Widom, J. Stat. Phys. 137, 825 (2009).

[6] K. A. Takeuchi and M. Sano, Phys. Rev. Lett. 104, 230601 (2010).

[7] C. Appert-Rolland, B. Derrida, V. Lecomte, and F. van Wijland, Phys. Rev. E 78, 021122 (2008).

[8] L. Bertini, A. De Sole, D. Gabrielli, G. Jona-Lasinio, and C. Landim, Phys. Rev. Lett. 94, 030601 (2005).

[9] T. Bodineau and M. Lagouge, J. Stat. Phys. 139, 201 (2010)

[10] A. Parmeggiani, T. Franosch, and E. Frey, Phys. Rev. Lett. 90, 086601 (2003).

[11] V. Popkov, A. Rákos, R. D. Willmann, A. B. Kolomeisky, and G. M. Schütz, Phys. Rev. E 67, 066117 (2003).
[12] G. M. Schütz, Europhys. Lett. 48, 623 (1999).

[13] T. M. Liggett, Stochastic Interacting Systems: Contact, Voter and Exclusion Processes (Springer, Berlin, 1999).

[14] G. M. Schütz, in Phase Transitions and Critical Phenomena, edited by C. Domb and J. Lebowitz, Vol. 19 (Academic, London, 2001), pp. 1-251.

[15] R. B. Stinchcombe, M. D. Grynberg, and M. Barma, Phys. Rev. E 47, 4018 (1993).

[16] M. D. Grynberg, T. J. Newman, and R. B. Stinchcombe, Phys. Rev. E 50, 957 (1994).

[17] G. M. Schütz, J. Phys. A: Math. Gen. 28, 3405 (1995).

[18] B. Derrida and J. L. Lebowitz, Phys. Rev. Lett. 80, 209 (1998).

[19] R. J. Harris and G. M. Schütz, J. Stat. Mech. Theor. Exp. (2007) P07020.

[20] R. Harris, A. Rákos, and G. M. Schütz, J. Stat. Mech. Theor. Exp. (2005) P08003.

[21] R. J. Harris, A. Rákos, and G. M. Schütz, Europhys. Lett. 75, 227 (2006).

[22] M. R. Evans and T. Hanney, J. Phys. A 38, R195 (2005). 